\title{
Differential contribution of transcription factors to Arabidopsis thaliana defense against Spodoptera littoralis
}

\author{
Fabian Schweizer, Natacha Bodenhausen ${ }^{\dagger}$, Steve Lassueur, Frédéric G. Masclaux and Philippe Reymond* \\ Department of Plant Molecular Biology, University of Lausanne, Lausanne, Switzerland
}

Edited by:

Corné M. Pieterse, Utrecht

University, Netherlands

Reviewed by:

Peer Schenk, The University of

Queensland, Australia

Roberto Solano, Centro Nacional de

Biotechnologia-Consejo Superior de

Investigaciones Cientificas, Spain

${ }^{*}$ Correspondence:

Philippe Reymond, Department of

Plant Molecular Biology, University of

Lausanne, Biophore Building, 1015

Lausanne, Switzerland.

e-mail: philippe.reymond@unil.ch

\section{${ }^{\dagger}$ Present address:}

Natacha Bodenhausen, Institute of

Microbiology, Swiss Federal Institute

of Technology Zurich, 8093 Zurich,

Switzerland.
In response to insect herbivory, Arabidopsis plants activate the synthesis of the phytohormone jasmonate-isoleucine, which binds to a complex consisting of the receptor COI1 and JAZ repressors. Upon proteasome-mediated JAZ degradation, basic helix-loop-helix transcription factors (TFs) MYC2, MYC3, and MYC4 become activated and this results in the expression of defense genes. Although the jasmonate (JA) pathway is known to be essential for the massive transcriptional reprogramming that follows herbivory, there is however little information on otherTFs that are required for defense against herbivores and whether they contribute significantly to JA-dependent defense gene expression. By transcriptome profiling, we identified $41 \mathrm{TFs}$ that were induced in response to herbivory by the generalist Spodoptera littoralis. Among them, nine genes, including WRKY18, WRKY40, ANAC019, ANAC055, ZAT10, ZAT12, AZF2, ERF13, and RRTF1, were found to play a significant role in resistance to $S$. littoralis herbivory. Compared to the triple mutant myc234 that is as sensitive as coi1-1 to herbivory, knockout lines of these nine TFs were only partially more sensitive to $S$. littoralis but, however, some displayed distinct gene expression changes at the whole-genome level. Data thus reveal that MYC2, MYC3, and MYC4 are master regulators of Arabidopsis resistance to a generalist herbivore and identify new genes involved in insect defense.

Keywords: Arabidopsis thaliana, Spodoptera littoralis, transcription factors, defense, MYC2, MYC3, MYC4

\section{INTRODUCTION}

During million years of coexistence, plants and insects have evolved different types of interactions. Some relationships like pollination are mutually beneficial, whereas the more common predator-host relationship is highly detrimental to plants (Walling, 2000). As a consequence, plants have developed several defense mechanisms to cope with insect attacks including physical barriers, the production of anti-digestive proteins, or toxic secondary metabolites (Howe and Jander, 2007). Most of these defenses are constitutive but are also highly inducible to minimize the cost of triggering defense in times of peace. In Arabidopsis and more generally in the Brassicaceae, the amino-acid derived glucosinolates (GS) have been extensively studied for their insect repellent/deterrent properties (Wittstock and Gershenzon, 2002; Halkier and Gershenzon, 2006). These compounds are generally stored as inactive molecules in the vacuole. Upon tissue or cell disruption, GS are catalyzed by myrosinases into active, highly toxic compounds including isothiocyanates, nitriles, and thiocyanates (Grubb and Abel, 2006; Halkier and Gershenzon, 2006).

Several studies have revealed that the plant hormone jasmonate (JA) is the main signal responsible for the activation of inducible defenses against arthropods and necrotrophic fungi (reviewed in Howe and Jander, 2007). In plants, herbivory triggers a burst of JA which leads to a massive transcriptional reprogramming and expression of defense genes (Reymond et al., 2000, 2004; Halitschke et al., 2001; de Vos et al., 2005; Devoto et al., 2005). The F-box protein COI1 was identified as a major component of the
JA-pathway, as coil-1 mutants were not responding to JA treatment (Xie et al., 1998) and were impaired in the expression of most JAand insect-inducible genes, including glucosinolate biosynthesisgenes (Reymond et al., 2004; Devoto et al., 2005; Mewis et al., 2006). Consequently, laboratory and field studies have shown that mutants compromised in JA biosynthesis or perception are highly affected in resistance against a wide range of insect herbivores (Howe et al., 1996; McConn et al., 1997; Baldwin, 1998; Stintzi et al., 2001; Li et al., 2004; Reymond et al., 2004; Paschold et al., 2007).

For years, the precise mode of JA perception had remained elusive until several studies provided evidence that COI1 itself, together with members of the JAZ family of repressors, forms a complex with jasmonate-isoleucine (JA-Ile), an amino-acid conjugated form of JA (Chini et al., 2007; Thines et al., 2007; Yan et al., 2007). Further work demonstrated that (+)-7-isoJasmonoyl-L-isoleucine is the natural and bioactive ligand of COI1-JAZ complexes (Fonseca et al., 2009). In the absence of JA-Ile, reflecting the state of non-attacked plants, JAZ proteins interact with the bHLH MYC2 transcription factor (TF) and NINJA, which in turn interacts with TPL to actively repress transcription of MYC2 target genes (TG; Pauwels et al., 2010). Upon herbivory, the accumulation and binding of JA-Ile to COI1 leads to ubiquitination and subsequent degradation of JAZs via the $26 \mathrm{~S}$ proteasome, allowing MYC2 to activate the expression of JA-responsive genes (Sheard et al., 2010; Pauwels and Goossens, 2011). 
While mechanisms of JA perception are being unveiled, relatively little is known on which transcription factors (TFs) are controlling such a massive transcriptional reprogramming and on which downstream genes are important for defense against herbivores. Although MYC2 has been shown to interact with JAZs and therefore potentially activate JA-responsive genes, several studies reported that contrary to coil-1 that is male sterile, myc2 alleles are fully fertile; moreover, they are only partially sensitive to exogenous JA and are only slightly more susceptible to insect herbivory than wild-type plants (Boter et al., 2004; Lorenzo et al., 2004; Dombrecht et al., 2007; Fernández-Calvo et al., 2011; Verhage et al., 2011). Recently, MYC2 was found to act additively with its closely related homologs MYC3 and MYC4 to control JA responses, including defense against herbivory (Fernández-Calvo et al., 2011). Indeed, a triple mutant myc2myc3myc4 (myc234) was as susceptible as coil-1 to the generalist herbivore Spodoptera littoralis and had a similar reduced expression of JA marker genes (Fernández-Calvo et al., 2011). Besides MYC factors, the insect-inducible Arabidopsis MYB102 was found to be necessary for defense against the specialist Pieris rapae (de Vos et al., 2006). Amyb102 mutant showed lower expression of defenseand cell wall-related genes. However, its connection with the JApathway was not examined (de Vos et al., 2006). Enhanced expression of MYB75 (PAP1), a gene that controls phenylpropanoid metabolism, by activation-tagging in Arabidopsis slowed growth of Spodoptera frugiperda, but the molecular mechanism of this response was not investigated (Johnson and Dowd, 2004). Similarly, heterologous expression of MYB12 in tobacco conferred increased resistance to Spodoptera litura and Helicoverpa armigera, presumably by the enhanced accumulation of flavonoids (Misra et al., 2010). Two WRKY TFs from Nicotiana attenuata, WRK3 and WRK6, were found to positively control the accumulation of JA-Ile and susceptibility to Manduca sexta, suggesting that these factors play a role upstream of the JA-pathway (Skibbe et al., 2008). Finally, GS biosynthesis is regulated by six R2R3-MYB TFs. MYB28, MYB29, and MYB76 control aliphatic-GS genes (Hirai et al., 2007; Gigolashvili et al., 2008; Sønderby et al., 2010), whereas MYB34, MYB51, and MYB122 control indole-GS genes (Gigolashvili et al., 2007). Overexpression of MYB51 in Arabidopsis impaired growth of Spodoptera exigua (Gigolashvili et al., 2007) whereas a myb28myb29 double mutant lacking aliphatic-GS was more susceptible to feeding by Mamestra brassicae (Beekwilder et al., 2008).

To identify novel TFs that respond to herbivory and to gain insight on their relative contribution to defense, we carried-out a transcriptomic search of insect-inducible TFs. We found nine TFs that had a significant effect on insect performance and analyzed insect-induced transcriptome changes in respective knockout lines. Our study reveals new players in Arabidopsis defense against a generalist herbivore and highlights the predominant role of MYC2, MYC3, and MYC4.

\section{MATERIALS AND METHODS \\ PLANT MATERIAL AND GROWTH CONDITIONS}

Arabidopsis thaliana Col-0 was the genetic background of all mutant lines used in this study. The following T-DNA insertion lines were obtained from the Nottingham Arabidopsis
Stock Center: erf13 (GK_121A12), nac019 (Salk_096295), nac055 (SALK_014331), wrky18 (SALK_093916), zat10 (SALK_054092), zat12 (SAIL_347_G03), azf2-1 (SALK_132562), rap2.6 (SAIL_1225_G09), rrtf1 (SALK_150614), myb44 (SALK_039074). Homozygous lines were selected by PCR and absence of transcription of the TG in mutant lines was confirmed by RTPCR. Specific forward and reverse primers were designed with SIGnAL T-DNA verification tool for all lines ${ }^{1}$. We generated nac019nac055 by crossing single mutants. Seeds of the triple mutant myc2myc3myc4 were a gift from Roberto Solano (Centro Nacional de Biotecnología-CSIC, Madrid, Spain). The coil1 (non-glabrous) mutant was obtained from Jane Glazebrook (University of Minnesota, St. Paul, MN, USA) and wrky40 and wrky18wrky40 mutants were obtained from Imre Somssich (Department of Plant Microbe Interactions, Max Planck-Institute for Breeding Research, Cologne, Germany).

Col-0 and mutant lines were stratified in water for 4 days at $4^{\circ} \mathrm{C}$. The $m y c 2 m y c 3 m y c 4$ mutant was stratified in water containing $0.1 \mathrm{mM}$ gibberellic acid to stimulate germination. Seeds were then transferred to pots containing potting compost. The coil-1 mutant was germinated on Murashige and Skoog medium (Sigma, Buchs, Switzerland) containing 3\% sucrose and $30 \mu \mathrm{M}$ JA and incubated under continuous light $\left(150 \mu \mathrm{mol} \mathrm{m}^{-2} \mathrm{~s}^{-1}\right)$ for 7 days in a growth chamber. Homozygous coil-1 mutants showing normal greening of leaves and no inhibition of root growth (Feys et al., 1994) were transferred to pots. Plants were grown in a growth chamber as previously described (Reymond et al., 2000).

\section{INSECT BIOASSAYS}

Spodoptera littoralis (Egyptian cotton worm) eggs were obtained from Syngenta (Stein, Switzerland) and were stored at $10^{\circ} \mathrm{C}$ until further use. Eggs were placed in a beaker covered with plastic film in an incubator $\left(26^{\circ} \mathrm{C}\right)$ for $2-3$ days to allow hatching. Larvae were then reared on Arabidopsis plants. For initial insect challenge, two to three fourth- or fifth-instar S. littoralis larvae were allowed to feed on 6-week-old plants for $4-5 \mathrm{~h}$ in a transparent plastic box in a growth chamber $\left(20^{\circ} \mathrm{C}, 65 \%\right.$ relative humidity, $100 \mu \mathrm{mol} \mathrm{m}^{-2} \mathrm{~s}^{-1}$, $10 / 14 \mathrm{~h}$ photoperiod) until approximately $20 \%$ of leaf area was removed. For each experiment, damaged leaf tissue from 12 challenged plants was harvested and immediately stored in liquid nitrogen. Leaves from 12 control, unchallenged plants were collected at the same time. For longer feeding experiments, newly hatched larvae (three for two plants) were allowed to feed continuously during 8 days until leaves were harvested. Microarray analyses with Col-0 and coil-1 plants were performed on at least three independent biological replicates.

For testing the susceptibility of TF mutants, 3-week-old plants were used. Forty newly hatched $S$. littoralis larvae were placed in a transparent plastic box containing 70 plants. After 8 days of feeding, larvae were collected and weighed on a precision balance (Mettler-Toledo, Greifensee, Switzerland) whereas plant tissues from control and treated plants were immediately stored in liquid nitrogen and used for microarray analyses. All experiments were repeated at least three times independently, except for erf13 and rrtfl mutants (two replicates).

${ }^{1}$ http://signal.salk.edu/tdnaprimers.2.html 


\section{MICROARRAY EXPERIMENTS AND DATA ANALYSIS}

For microarray analysis, total RNA of plant tissues was extracted, reverse-transcribed, and processed according to a previously published procedure (Bodenhausen and Reymond, 2007). Labeled probes were hybridized onto CATMAv4 microarrays containing 32,998 Arabidopsis gene-specific tags and gene-family tags (Sclep et al., 2007). Hybridization and scanning have been described previously (Reymond et al., 2004). Data normalization and statistical analyses including false-discovery rate (FDR) correction were carried-out using an interface developed at the University of Lausanne [Gene Expression Data Analysis Interface (GEDAI; Liechti et al., 2010)]. Hierarchical clustering of microarray data as well as gene node heights calculations were done with Multi experiment viewer software ${ }^{2}$ using the default options. Microarray data have been submitted to ArrayExpress database under accession E-MTAB- $1418^{3}$.

\section{QUANTITATIVE RT-PCR}

Leaf samples from 5 to 10 plants were harvested and pooled after $48 \mathrm{~h}$ of herbivory by first-instar S. littoralis larvae. Tissue samples were ground in liquid nitrogen and total RNA was extracted using RNeasy Plant Mini Kit and treated with DNaseI (Qiagen, Hombrechtikon, Switzerland). Afterward, cDNA was synthesized from $1 \mu \mathrm{g}$ of RNA using M-MLV reverse transcriptase (Invitrogen, Zug, Switzerland) in a final volume of $25 \mu \mathrm{l}$ and subsequently diluted fourfold with water. Gene-specific primers were designed to produce amplicons between 80 and $120 \mathrm{bp}$. Primer efficiencies (E) were evaluated by five-step dilution regression. Quantitative real-time PCR (qRT-PCR) was performed using Brilliant II Fast SYBR-Green qRT-PCR Master Mix. Reactions were done in a final volume of $25 \mu \mathrm{l}$ containing $12.5 \mu \mathrm{l}$ of $2 \times$ SYBR, $3.75 \mu \mathrm{l}$ of ROX (1/5000 dilution), $4.25 \mu \mathrm{l}$ of RNAse-free water, $2.5 \mu \mathrm{l}$ of primer mix (each primer at $1 \mu \mathrm{M}$ ), and $2 \mu \mathrm{l}$ of cDNA. A Mx3000P realtime PCR instrument (Agilent, Morges, Switzerland) was used with the following program: $95^{\circ} \mathrm{C}$ for $5 \mathrm{~min}$, then 40 cycles of $10 \mathrm{~s}$ at $95^{\circ} \mathrm{C}, 20 \mathrm{~s}$ at $55^{\circ} \mathrm{C}$, and $30 \mathrm{~s}$ at $60^{\circ} \mathrm{C}$. Values were normalized to the house-keeping gene ACTIN8. The expression level of a TG was normalized to the reference gene (RG) and calculated as Normalized Relative Quantity (NRQ) as follows: $\mathrm{NRQ}=E^{\mathrm{CtRG}} / E^{\mathrm{CtTG}}$. Each experiment was repeated three times independently.

\section{GLUCOSINOLATE ANALYSIS}

For GS extraction, seven 3-week-old plants were challenged for $48 \mathrm{~h}$ with two neonate $S$. littoralis larvae per leaf. Unchallenged plants were used as controls. Samples from four biologically independent replicates were analyzed. Extraction method, UHPLCQTOFMS measurements and analysis have been recently described (Glauser et al., 2012).

\section{RESULTS}

\section{IDENTIFICATION OF INSECT-INDUCED TRANSCRIPTION FACTORS}

To identify novel TFs that are involved in the response to herbivory, we reasoned that some of these factors might be themselves subjected to transcriptional regulation. We therefore performed

\footnotetext{
${ }^{2}$ http://www.tm4.org/mev/

${ }^{3}$ http://www.ebi.ac.uk/arrayexpress/
}

a whole-genome microarray analysis of Arabidopsis plants challenged with the generalist $S$. littoralis and searched for TF genes that were robustly induced by herbivory. We collected RNA from several independent replicates after $5 \mathrm{~h}$ of feeding with fourth-fifth instar larvae and after 8 days of feeding with neonate larvae and analyzed the transcriptome using Arabidopsis CATMA microarrays (Sclep et al., 2007). In addition, to evaluate the role of the JA-pathway in regulating these TFs, we used coil-1 plants in the same experimental set-up. Induced genes were defined as genes with a mean expression ratio $\geq 2$ in Col-0 (adjusted $P$ value $<0.05)$. Based on TAIR annotation ${ }^{4}$, we identified 41 TFs that were significantly up-regulated by $S$. littoralis herbivory (Table $\mathrm{S} 1$ in Supplementary Material). Clustering microarray data of Col0 and coil-1 plants showed that most TFs were not or much less induced in coil-1 plants, suggesting that they depend on a functional JA-pathway (Figure 1; Table S1 in Supplementary Material). Induced TFs belonged to different classes, including for example several ERF/AP2, bHLH, MYB, WRKY, Zinc-fingers, and NAC factors.

\section{INSECT PERFORMANCE ON TF KNOCKOUT LINES}

Larval growth can be used as an outcome of plant defense ability against herbivores. To assess whether the newly identified insect-induced TFs where involved in defense, we obtained TDNA knockout lines and challenged them with insects. For this assay, 3-week-old plants were subjected to feeding by neonate S. littoralis for 8 days. Among the 41 insect-induced TFs, some were already known to be involved in defense against herbivory (MYC2, MYB34, MYB75) and were not tested further. For the other candidates, we obtained 11 homozygous mutant lines, of which nine showed a significantly higher growth of S. littoralis larvae (Figure 2; Table 1). Larval weight was between 27\% (erf13) and $66 \%$ (zat12) higher on mutant than on wild-type plants, but this was less pronounced than on coil-1 or myc234 plants (>300\%; Table 1). Interestingly, all sensitive TF mutants belonged to unrelated gene families like bHLH ( myc234), WRKY (wrky18, wrky40), NAC (nac019, nac055), zinc-finger (zat10, zat12, azf2-1), and ERF/AP2 (erf13 and rrtf1). For some closely related TFs like WRKY18, WRKY40, and NAC019, NAC055, the respective double mutants were also tested. Noteworthy, although both single mutants were significantly more sensitive to herbivory, none of the double mutants showed an additive effect on larval growth (Figure 2). A plausible explanation could be that these factors form heterodimers and control the same sets of defense genes.

\section{EXPRESSION OF JA MARKER GENES}

Several studies have shown that the JA-pathway positively controls the expression of at least two distinct sets of genes. Herbivory leads to a burst of JA which activates the expression of genes like JAZ10 and VSP2 (Reymond et al., 2004; Yan et al., 2007). In response to necrotrophic fungi, plants produce JA and ethylene (ET), which together turn on a set of genes including PDF1.2 and ORA59 (Manners et al., 1998; Penninckx et al., 1998; Pré et al., 2008). To test the involvement of insect-responsive TFs in the activation

\footnotetext{
${ }^{4}$ www.arabidopsis.org
} 


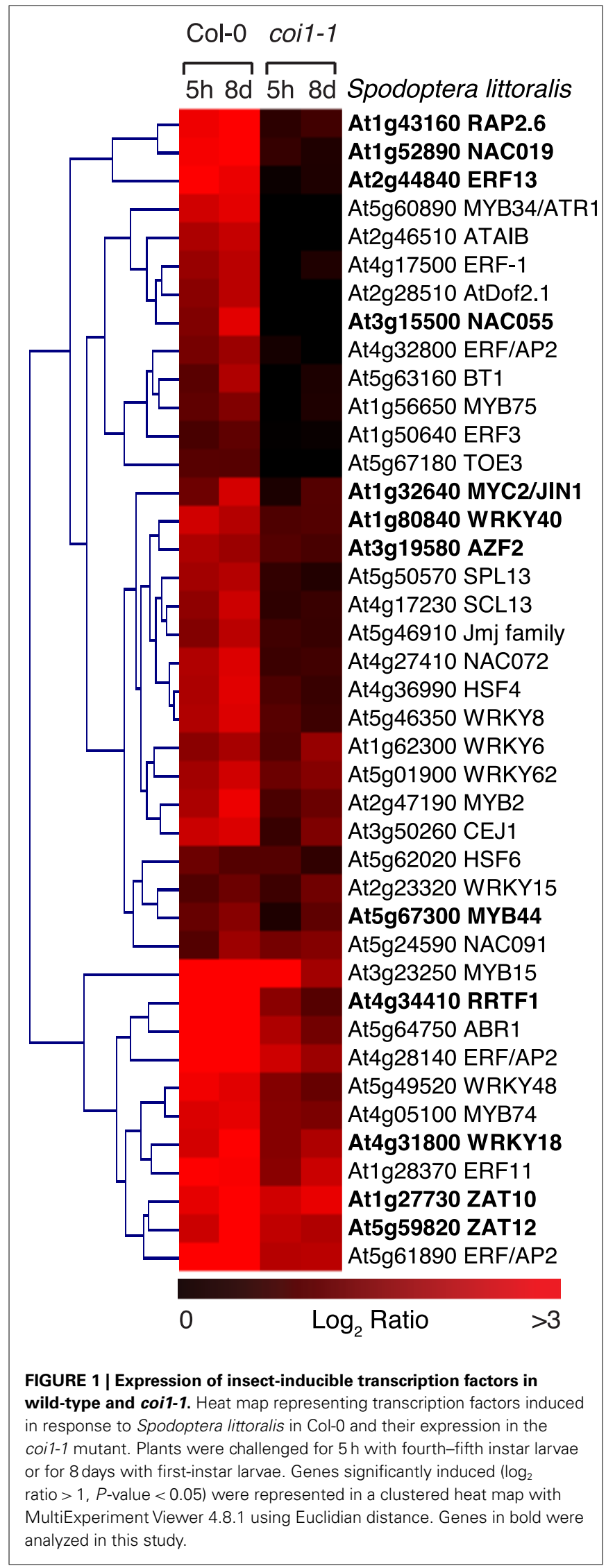

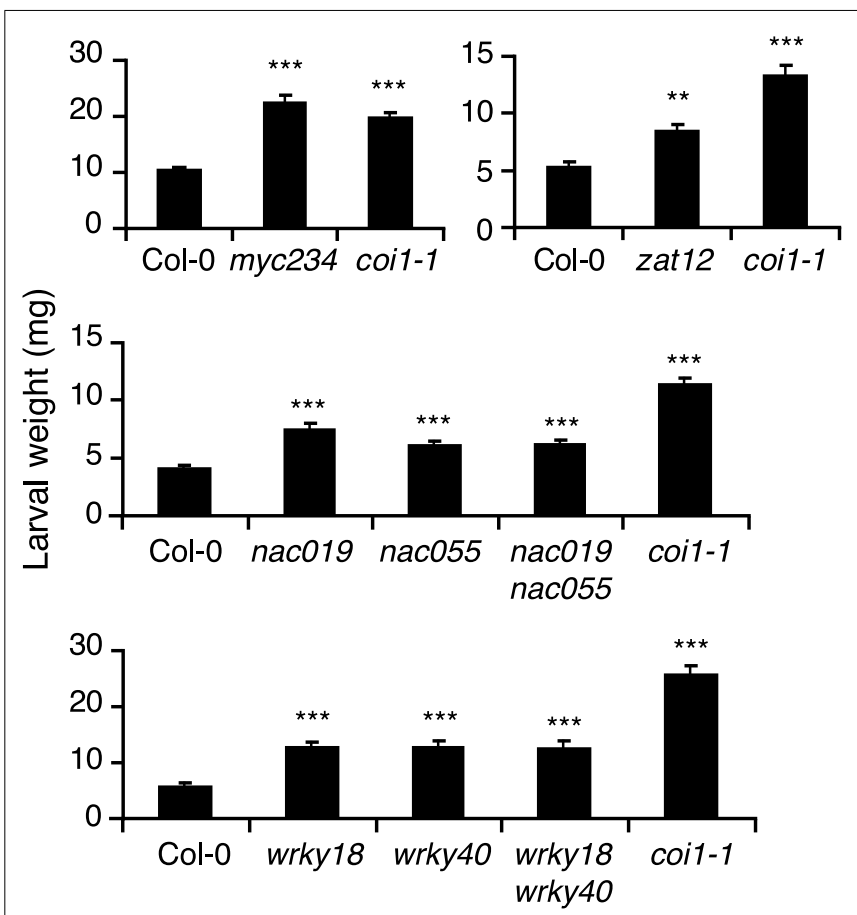

FIGURE 2 | Insect performance on transcription factor mutants. Freshly hatched $S$. littoralis larvae were placed on each genotype and larval weight (mean \pm SE) was measured after 8 days of feeding. Asterisks indicate statistically significant differences between mutant plants and Col-0 (Student's $t$-test, ${ }^{*} P<0.05,{ }^{*} P<0.01,{ }^{*}{ }^{*} P<0.001$ ). Similar results were observed in at least three independent replicate experiments.

of these two branches of the JA-pathway, we monitored VSP2 and PDF1.2 expression in mutant lines by qRT-PCR. VSP2 induction by $S$. littoralis was significantly reduced in nac019, nac019nac055, wrky 18, wrky40, wrky 18 wrky 40 mutants, although to a lesser extent than in coil-1, but was not affected in nac055, zat10, zat12, and erf13 mutants (Figure 3). Interestingly, up-regulation of PDF1.2 was higher in all nac mutants, as well as in wrky18, wrky18wrky40, zat10, and zat12, than in Col-0. On the contrary, PDF1.2 expression was abolished in coil-1 (Figure 3). Noteworthy, such opposite expression of PDF1.2 was previously observed between myc234 and coil-1 in response to JA treatment (Fernández-Calvo et al., 2011). Our results suggest that increased insect susceptibility of some TF mutant lines can be explained by a reduced activation of the JA-pathway that leads to the accumulation of anti-insect proteins, including VSP2.

\section{WHOLE-GENOME ANALYSIS OF TF MUTANTS}

To gain more insight on the role of insect-induced TFs on downstream gene expression, we carried-out microarray analyses with mutant lines that showed a higher sensitivity to S. littoralis. As controls for highly sensitive mutants, we included coil-1 and myc234. $S$. littoralis larvae were allowed to feed for 8 days on Col-0 and mutant plants, then RNA was extracted and hybridized to CATMA microarrays. As expected, the majority of genes induced by herbivory in Col-0 were JA-dependent and thereby were not induced in coil-1 (Figure 4A). In accordance with their similar insect 
Table 1 | Insect performance on transcription factor mutants.

\begin{tabular}{lll}
\hline Mutant & AGI & Relative weight \\
\hline coi1-1 & At2g39940 & $3.00 \pm 0.23^{* * *}$ \\
myc234 & At1g32640/At5g46760/At4g17880 & $3.10 \pm 0.42^{* *}$ \\
nac019 & At1g52890 & $1.50 \pm 0.14^{* *}$ \\
nac055 & At3g15500 & $1.38 \pm 0.17^{* *}$ \\
nac019nac055 & At1g52890/At3g15500 & $1.40 \pm 0.24^{* *}$ \\
zat10 & At1g27730 & $1.45 \pm 0.28^{* *}$ \\
zat12 & At5g59820 & $1.66 \pm 0.05^{* *}$ \\
azf2-1 & At3g19580 & $1.54 \pm 0.07^{* *}$ \\
wrky18 & At4g31800 & $1.57 \pm 0.20^{* *}$ \\
wrky40 & At1g80840 & $1.54 \pm 0.38^{* *}$ \\
wrky18wrky40 & At4g31800/At1g80840 & $1.46 \pm 0.13^{* * *}$ \\
rrtf1-1 & At4g34410 & $1.30 \pm 0.08^{* * *}$ \\
erf13 & At2g44840 & $1.27 \pm 0.13^{* * *}$ \\
rap2.6 & At1g43160 & $1.32 \pm 0.22$ n.s. \\
myb44 & At5g67300 & $1.00 \pm 0.11$ n.s. \\
\hline
\end{tabular}

Relative weight corresponds to the mean weight of neonate $S$. littoralis larvae feeding on 3-week-old mutants for 8 days divided by the mean weight of larvae feeding on Col-O. Values ( $\pm S E$ ) are the mean of several replicates (mutants $n \geq 3 ;$ myb44 $n=2 ;$ Col-0 $n=17$ ). Asterisks indicate $P$-value (n.s. not significant; ${ }^{*}<0.05 ;{ }^{* *}<0.01 ;{ }^{* *}<0.001$; Nested ANOVA).

susceptibility, myc234 and coil-1 showed a very similar expression profile, corroborating the additive role of MYC2, MYC3, and MYC4 as general transcriptional regulators acting directly downstream of COI1 to control the expression of JA-responsive genes (Fernández-Calvo et al., 2011).

Although other TF mutants showed an overall expression pattern that was more similar to Col-0, they anyhow displayed altered profiles (Figure 4A). A correspondence analysis where the weight distance between different experiments is indicative of their relative similarity indicated that coil-1 and myc234 expression profiles form a distinct subgroup that is distant from a second subgroup containing Col-0 and all TF mutant profiles (Figure 4B). In this second subgroup, wrky 18 and wrky 40 mutants formed a clearly separated branch, as well as erf13 and rrtf1, two members of the B3 sub-family of ERF/AP2 TFs that clustered together, whereas nac019, nac055, zat10, zat12, and azf2-1 were more similar to Col-0 (Figure 4B).

Although coil-1 and myc234 expression profiles were globally similar, we could however detect significant differences in the expression of several genes. We observed that some COI1-dependent genes were normally expressed in myc234, as for instance PDF1.2 and a myrosinase-associated protein (Atlg54010), or showed a reduced induction, as for instance VSP2, CORI3, and MYB75 (Table 2; Table S2 in Supplementary Material). Thus, the distance separating transcriptomes of coil-1 and $m y c 234$ on the cluster (Figure 4B) probably reflects the expression changes of such genes.

A careful examination of the expression profiles of nac019, nac055, nac055nac019, zat10, zat12, azf2-1, rrtf1, and erf13 mutants did not allow to identify candidate defense genes that could easily explain the susceptibility to $S$. littoralis. As illustrated

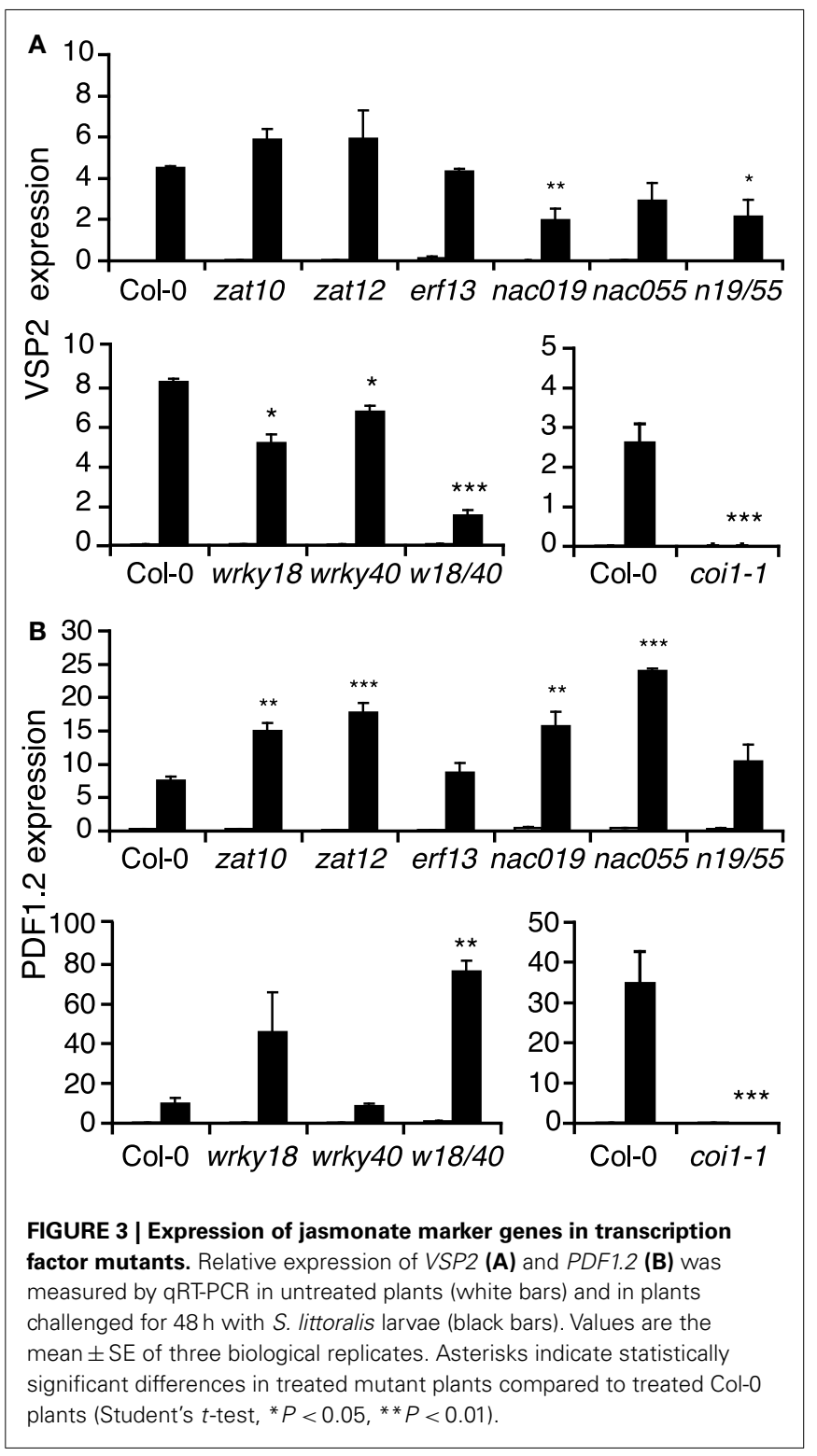

by the clustering of TF mutant expression profiles with Col-0 (Figure 4B), the large majority of insect-inducible genes were still up-regulated in the mutants (Table S2 in Supplementary Material). However, consistent with the fact that wrky mutants formed a distinguishable group in the cluster, they showed a partially reduced expression of genes from several pathways including general defense (protease inhibitors), JA-biosynthesis (LOX2), GS biosynthesis (MYB34, CYP79B3), and breakdown (TGG2), and phenylpropanoid biosynthesis (DFR, CHS; Table S2 in Supplementary Material). To test whether the transcriptional change in GS biosynthesis-genes could effectively alter GS biosynthesis, we quantified GS in wrky18wrky40 by UHPLC-QTOFMS (Glauser et al., 2012). Analysis of the most abundant GS showed that, in response to $S$. littoralis, wrky18wrky40 accumulated significantly more methylthio-GS (4MTB, 7MTH, 8MTO), less methylsulfylGS (4MSOB, 8MSOO), and less indole-GS (I3M, 1MO-I3M) than 

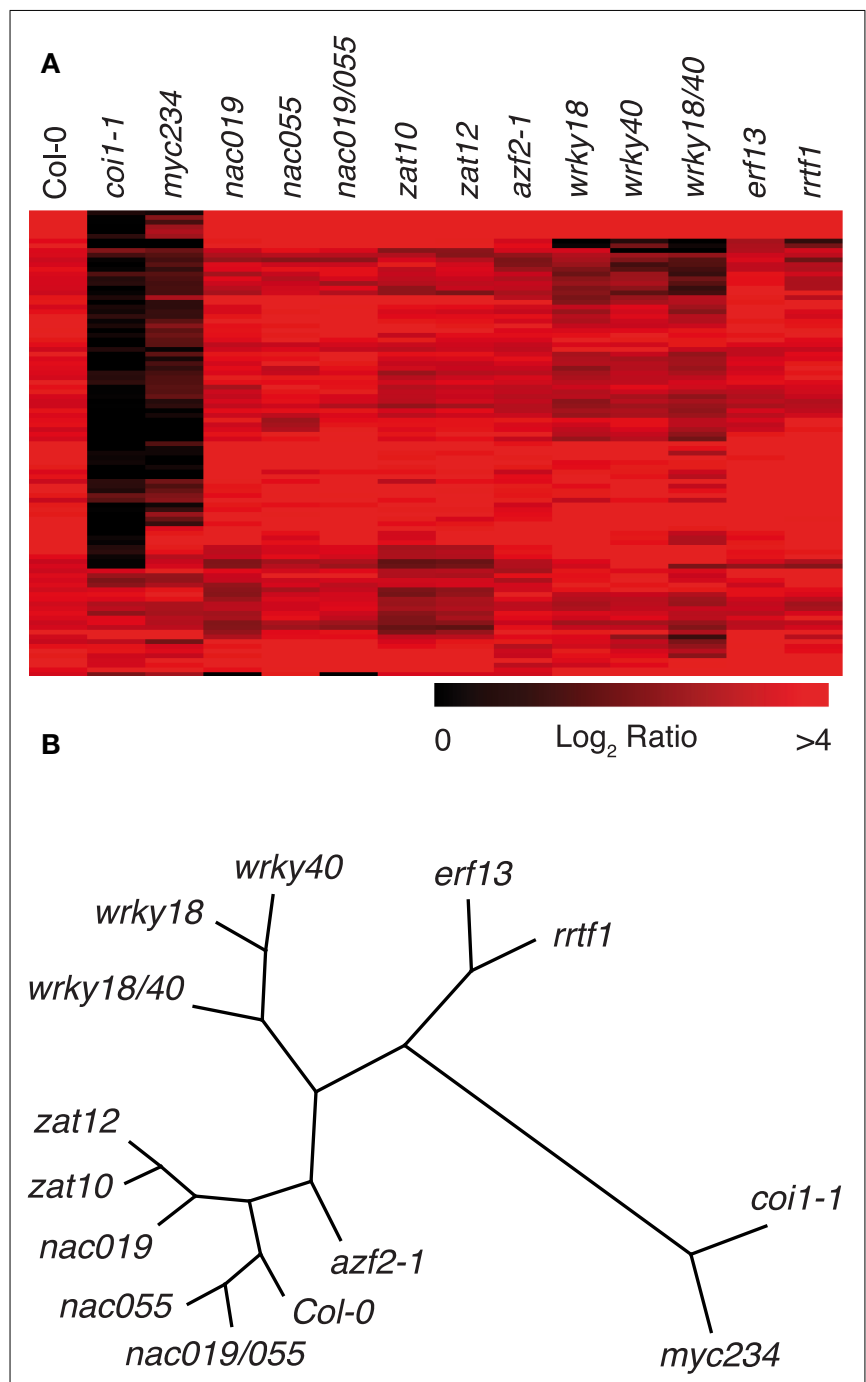

FIGURE 4 | Whole-genome expression profile of transcription factor mutants. (A) Heat map clustering the 100 most highly induced genes in Col-0 plants after 8 days of insect feeding and their respective expression in mutant plants. Heat map was created with MultiExperiment Viewer 4.8.1. (B) Correspondence analysis of expression profiles including all insect-induced genes $\left(\log _{2}\right.$ ratio $>1, P$-value $\left.<0.05 ; n=874\right)$. Clustering and node length calculations were performed with MultiExperiment Viewer 4.8.1 and represented as unrooted tree in Treeview 1.6.6 (http://taxonomy.zoology.gla.ac.uk/rod/treeview.html).

Col-0 (Figure 5A). Thus, although the total GS amount between wrky18wrky40 and Col-0 was similar, these qualitative differences could contribute to the increased insect susceptibility of the mutant.

To test the involvement of WRKY18 and WRKY40 in the phenylpropanoid pathway, we analyzed three genes involved in the last steps of anthocyanins and flavonols biosynthesis. Expression analysis by qRT-PCR showed clearly that DFR, LDOX, and $3 \mathrm{GT}$ were strongly induced by herbivory in Col-0, whereas no significant induction could be observed in wrky18wrky40 (Figure 5). The insect sensitive phenotype of wrky18wrky 40 could therefore be explained in part by a reduced accumulation of metabolites from the phenylpropanoid pathway.

\section{EXPRESSION OF INSECT-INDUCIBLE TFs IN coi1-1 AND myc234}

We found that some TF mutants show an altered expression of JA marker genes but that this was not as severe as in coil-1 and myc234 plants (Figure 3, Table S2 in Supplementary Material). We thus wondered whether this regulation was done through the COI1/MYC234 signaling module or whether these TFs were independent modulators of defense gene expression. To address this hypothesis, we analyzed the expression of nine TFs whose mutants were more sensitive to insects in Col-0, coil-1, and myc234. All tested TFs were highly induced in response to $S$. littoralis, validating the microarray data (Figure 6). Moreover, TF expression pattern in coil-1 and myc234 could be separated into two different types of responses. First, NAC019, NAC055, ERF13, and RRTF1 were all significantly less induced in coil-1 and myc234 than in Col0 (Figure 6). Interestingly, NAC019, NAC055, ERF13, and RRTF1 were barely induced in coil-1 mutants but did still show a slight induction in myc234. Taken together, it seems that these genes depend on a functional JA-pathway and are thus not induced in coil-1, whereas a redundant MYC or other TFs might contribute to their partial expression in $m y c 234$. The second group included genes whose expression was still induced in coil-1 and myc234, but somewhat reduced when compared to Col-0 (Figure 6). Induction of ZAT10, ZAT12, AZF2, WRKY18, and WRKY40 was reduced in coil-1 and myc234 compared to Col-0, although the difference with Col-0 was only statistically significant for ZAT12 in coil-1 and $A Z F 2$ in myc234.

Previous reports have shown that MYC2, MYC3, and MYC4 bind preferentially to G-box and G-box like sequences in the promoter of TGs (Dombrecht et al., 2007; Fernández-Calvo et al., 2011; Godoy et al., 2011). We further investigated whether there was a correlation between TF expression patterns and the presence of MYC2 binding cis-elements in their respective promoters. Nearly all promoters contained G-box and G-box like sequences, indicating that they might be direct targets of MYCs (Table 3). The exception was WRKY18 and ERF13 that did not contain any Gbox element. Since ERF13 expression was strongly dependent on COI1 and MYC2, MYC3, MYC4 (Figure 6), this gene must thus be indirectly controlled by MYCs. Taken together, our findings suggest that enhanced insect performance on coil-1 and myc234 is explained in part by a reduced expression of downstream TFs that regulate the expression of defense genes.

\section{DISCUSSION}

During insect herbivory, plants induce about 1000 genes, of which roughly $65 \%$ are regulated by the JA-pathway (Halitschke et al., 2001; Reymond et al., 2004; de Vos et al., 2005; Devoto et al., 2005). Following JA-Ile perception by COI1, repression of MYC2, MYC3, and MYC4 by JAZs is released allowing the transcription of defense genes. Consequently, coil-1 and myc234 mutants display a strong susceptibility to herbivory (Fernández-Calvo et al., 2011). In order to identify novel TFs involved in plant response to herbivory, we performed a whole-genome expression analysis and found 41 TFs that were robustly induced after short- or long-term feeding by the generalist $S$. littoralis. From these, we obtained 11 
Table 2 | List of insect-induced genes.

Expression ratio $\left(\log _{2}\right)$

\section{Description}

TI1, trypsin inhibitor

RD20, calcium-binding protein

Protease inhibitor (LTP)

Aldo/keto reductase

CAD8, cinnamyl-alcohol dehydrogenase

Strictosidine synthase

Protease inhibitor (LTP)

Protease inhibitor

PRX52, peroxidase

Trypsin and protease inhibitor

FAD-binding berberine family protein

FAD-binding berberine family protein

PDF1.2, plant defensin

Myrosinase-associated protein

Oxidoreductase, 2OG-Fe(II) oxygenase

GCN5-related N-acetyltransferase (GNAT)

Palmitoyl protein thioesterase

VSP2, acid phosphatase

CORI3, cystine lyase

BAM5, beta-amylase

PAP1 (MYB75), transcription factor

Terpene synthase/cyclase,

Oxidoreductase, 2OG-Fe(II) oxygenase

Jacalin lectin

TSA1, calcium-binding protein

SSRP1, DNA-binding protein

GOLS1, galactinol synthase

Expressed protein

ARGAH2, arginase

DHAR1, dehydroascorbate reductase

Cysteine proteinase

ILL6, IAA amino-acid conjugate hydrolase

$\mathrm{AOC1}$, allene oxide cyclase

LOX3, lipoxygenase

Protein kinase

Oxidoreductase, 2OG-Fe(II) oxygenase

jacalin lectin,

FAMT, farnesoic acid methyl transferase

VSP1, acid phosphatase

JAZ10

MBP2, myrosinase-binding protein

$M B P 1$, myrosinase-binding protein

TRAF-like family protein

UTR3, UDP-galactose transporter

Trypsin and protease inhibitor

O-methyltransferase

TRAF-like family protein

AT14A, transmembrane protein

AT14A, transmembrane protein

PGL5, 6-phosphogluconolactonase
AGI

At2g43510

At2g33380

At4g12500

At2g37770

At4g37990

At1g74010

At4g12490

At2g38870

At5g05340

At1g73260

At4g20860

At2g34810

At5g44420

At1g54010

At5g05600

At2g39030

At4g 17470

At5g24770

At4g23600

At4g15210

At1g56650

At1g61120

At2g38240

At1g52000

At1g52410

At3g28730

At2g47180

At4g02360

At4g08870

At1g19570

At4g11320

At1g44350

At3g25760

At1g17420

At4g10390

At3g55970

At2g39330

At3g44860

At5g24780

At5g13220

At1g52030

At1g52040

At5g26260

At1g14250

At1g73325

At1g76790

At3g28220

At3g28300

At3g28290

At5g24420 coi1-1

myc234

\section{Col-0}

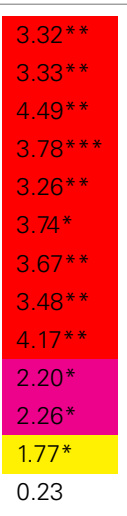

0.77

0.03

$-0.34$

$-0.05$

0.81

0.55

$-0.54$

0.58

0.07

$-0.24$

$-0.39$

0.41

0.77

0.85

0.60

$-0.15$

0.11

$-0.06$

$-0.56$

$-0.18$

0.87

0.21

0.14

$-0.57$

$-1.17$

0.59

$-0.33$

0.33

0.39

0.40

$-0.42$

$-0.19$

$-1.09$

$-1.12$

$-1.06$

$-1.12$

$-0.83$

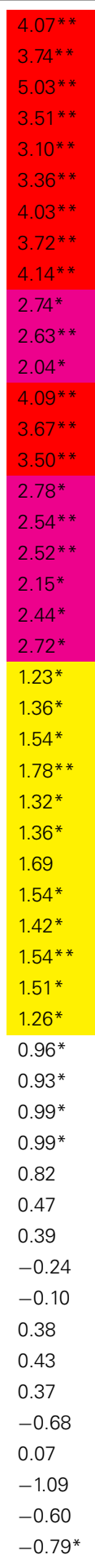

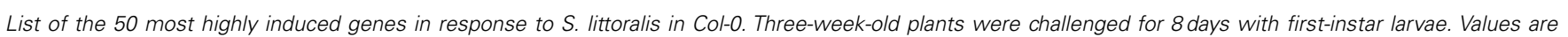
calculated from several independent biological replicates (Col-0, myc234 $n=4 ;$ coi1-1: $n=3$ ). Ratios are color-coded according to intensity: yellow (from 1 to 2 ), magenta (from 2 to 3), red (>3). Asterisks indicate adjusted P-value $\left(^{*}<0.05 ;{ }^{*}<0.01 ; * * * 0.001\right.$ ). 


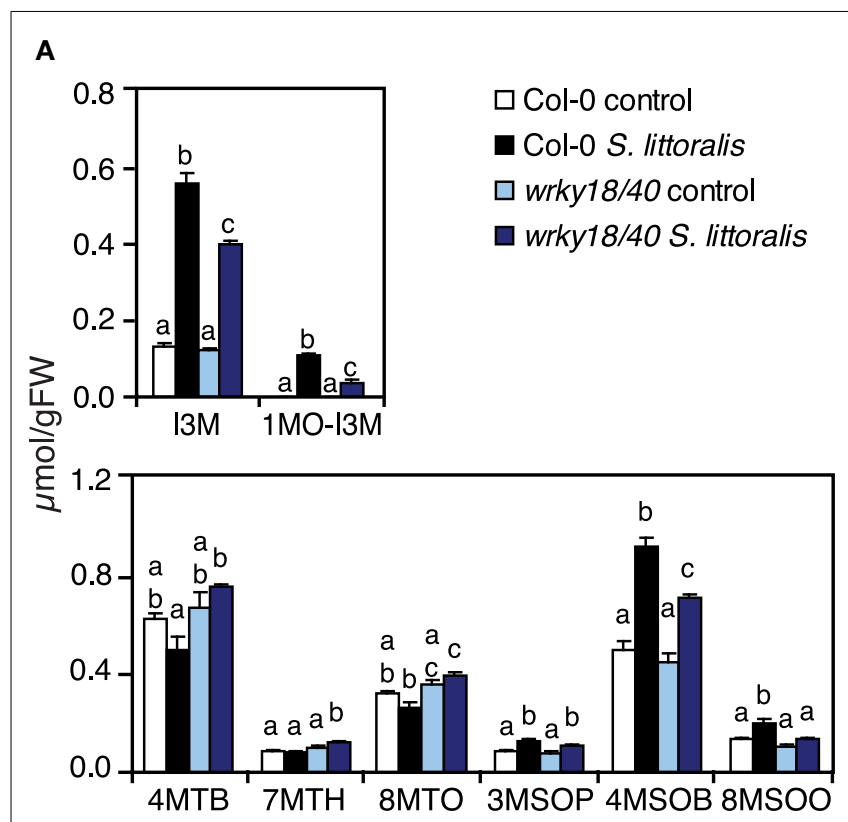

B

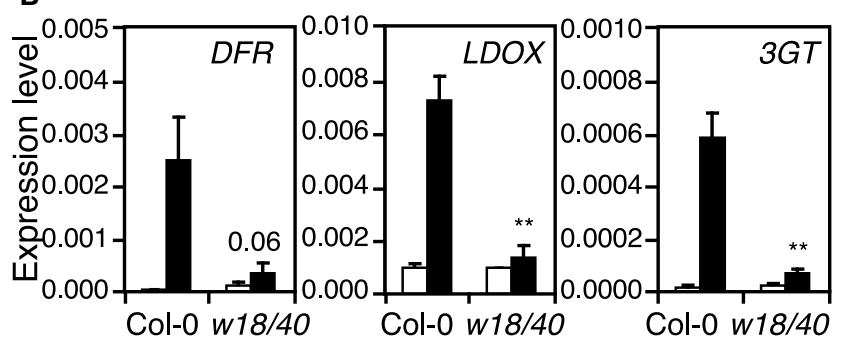

FIGURE 5 | Quantification of glucosinolates and expression of phenylpropanoid pathway genes in wrky18wrky40 mutant. (A) Levels of eight glucosinolates were quantified in Col-0 and wrky18wrky40 double mutant. Plants were challenged for 2 days with $S$. littoralis larvae. Unchallenged plants were used as controls. Values are the mean ( \pm SE) of four biological replicates. Bars with different letters differ at $P<0.05$ (Tukey's HSD test). 4MTB, 4-Methylthiobutyl-GS; 7MTH,

7-Methylthioheptyl-GS; 8MTO, 8-Methylthiooctyl-GS; 3MSOP,

3-Methylsulfinylpropyl-GS; 4MSOB, 4-Methylsulfinylbutyl-GS; 8MSOO,

8-Methylsulfinyloctyl-GS; I3M, Indol-3-ylmethyl-GS; 1MO-I3M,

1-Methoxyindol-3-ylmethyl-GS. (B) The wrky18wrky40 mutant shows altered expression of phenylpropanoid pathway genes DFR, LDOX, and $3 G T$ in response to herbivory. Relative expression was measured by qRT-PCR in untreated plants (white bars) and in plants challenged for $48 \mathrm{~h}$ with $S$. littoralis larvae (black bars). Values are the mean $( \pm S E)$ of three replicate experiments. Asterisks indicate statistically significant differences in treated wrky18wrky 40 plants compared to treated Col-0 plants (Student's $t$-test, ${ }^{*} P<0.05,{ }^{*} P<0.01,{ }^{*}{ }^{*} P<0.001$ ).

mutants of which nine were found to increase insect performance. However, mutation in none of these TFs was able to phenocopy the severe susceptibility observed with coil-1 and myc234, suggesting that these factors only partially contribute to insect defense. One explanation could be that these TFs are downstream targets of MYCs and that they regulate subsets of defense genes. However, analysis of their expression in coil-1 and myc234 revealed that this was not always the case. Whereas NAC019, NAC055,
$E R F 13$, and $R R T F 1$ induction by herbivory was clearly dependent on COI1 and MYC2/MYC3/MYC4, expression of other TFs was not, or only partly, affected in the mutants. We thus propose a model where groups of TFs activates defense gene expression in JA-dependent and JA-independent manner (Figure 7). For the JA-dependent pathway, MYC2/MYC3/MYC4 play a quantitatively important role by directly activating defense genes or by activating downstream TFs. In parallel, a JA-independent pathway triggers WRKYs and Zinc-finger TFs expression to provide additional defense. These findings might however represent only a fraction of all TFs involved in defense against herbivory. First, we could only obtain 11 confirmed mutants and the implication of the other insect-induced TFs should be tested. Second, it is also possible that important TFs are not induced by herbivory. For example, expression of MYC3 and MYC4 is not up-regulated by JA treatment (Fernández-Calvo et al., 2011).

Although myc234 and coi1-1 were equally sensitive to herbivory, confirming previous observations (Fernández-Calvo et al., 2011), we found that their expression profile was not identical and several potential defense genes were induced in Col-0 and myc234 but not in coil-1. This suggests that other yet unknown TFs are targets of the COI1-JAIle-JAZs signaling module. Since the JA-pathway is also crucial for defense against necrotrophic fungi (Thomma et al., 2000; Thaler et al., 2004), it is plausible to postulate that specific TFs are involved in this response. Recently, it was shown that JAZs bind to ethylene-stabilized TFs EIN3 and EIL1 to repress the activation of downstream genes ERF1 and PDF1.2 (Zhu et al., 2011). Interestingly, PDF1.2 induction by $S$. littoralis was larger in myc234, wrky18wrky40, zat10, zat12, nac019, and nac055 than in Col-0, whereas it was abolished in coil-1, indicating that this branch of the JA-pathway, that requires also ethylene, might be under the negative regulation of the MYC2/MYC3/MYC4 branch. It would be interesting to test the response of $m y c 234$ and TF mutants to necrotrophic fungi.

Global expression profiles of most TF mutants in response to herbivory displayed a moderate change compared to Col-0, whereas coil-1 or myc234 had a marked reduction of defense gene expression. However, these mutants displayed a significant increased sensitivity to $S$. littoralis, indicating that each TF is controlling the expression of important defense genes. One interpretation could be that mutation in these TFs strongly affected the basal expression of defense genes and, whereas the expression ratios were similar between wild-type and mutant plants, the absolute expression level in insect-treated plants might be considerably lower. However, an analysis of expression levels of the most highly insect-induced genes did not show a drastic difference between Col-0 and TF mutants (not shown). Alternatively, the enhanced susceptibility of TF mutant plants might be due to a small but general reduction of defense gene expression. Finally, the downregulation of a few specific genes that have a strong impact on defense could also explain these results. Future research will be required to elucidate which hypothesis is true.

Results from whole-genome expression profiles placed wrky mutants in a distinct subgroup. Induction of the anti-insect protein VSP2 (Liu et al., 2005) was partially reduced in wrky18, wrky40, and more strongly in wrk18wrky40 mutants. The expression of genes related to several pathways was also affected, in 

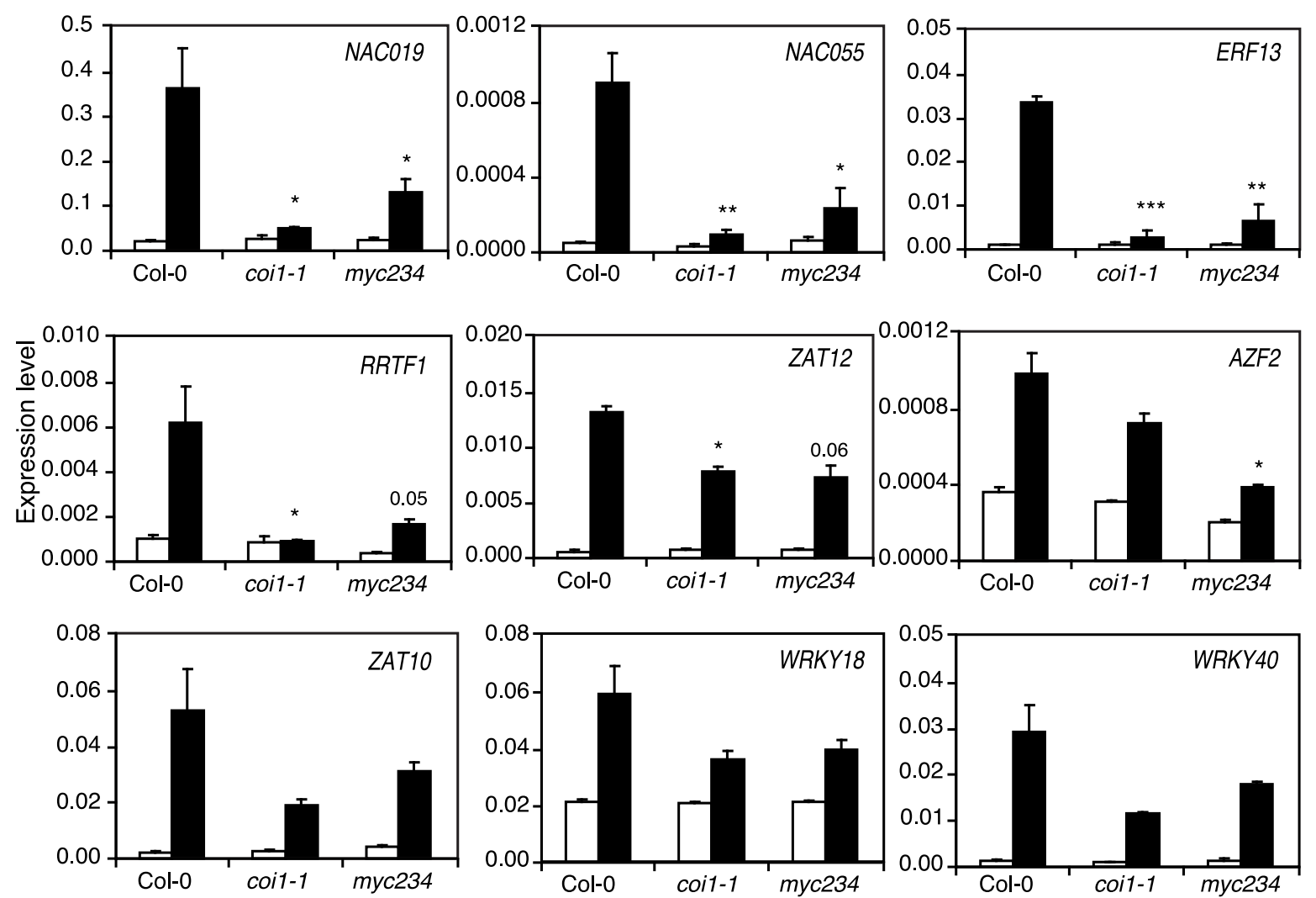

FIGURE 6 | Expression of insect-inducible transcription factors in coi1-1 and $\boldsymbol{m y c 2 3 4}$. Expression of TFs was measured by qRT-PCR in untreated plants (white bars) and in plants challenged for $48 \mathrm{~h}$ with $S$. littoralis larvae (black bars). Values are the mean $( \pm S E)$ of three biological replicates. Asterisks indicate statistically significant differences in treated plants compared to Col-0 (Student's $t$-test, ${ }^{*} P<0.05,{ }^{*} P<0.01,{ }^{* *} P<0.001$ ).
Table 3 | MYC-binding sites in the promoter of insect-induced TFs.

\begin{tabular}{|c|c|c|c|c|c|c|c|c|c|c|c|}
\hline AGI & Gene & 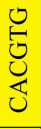 & 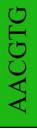 & 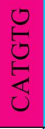 & 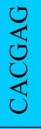 & 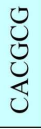 & $\begin{array}{l}0 \\
0 \\
0 \\
ن \\
ن \\
ن\end{array}$ & $\stackrel{\circlearrowright}{\stackrel{E}{\rightleftarrows}}$ & 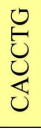 & $\begin{array}{l}0 \\
0 \\
0 \\
0 \\
0\end{array}$ & sum \\
\hline Atlg52890 & ANAC019 & 4 & & & 1 & & & & & & 5 \\
\hline At3g 15500 & ANAC055 & 2 & & 1 & 1 & & & 1 & & & 5 \\
\hline At2g44840 & ERF13 & & & & & & & & & & 0 \\
\hline At4g34410 & RRTF1 & 2 & 1 & 2 & & & & & & & 5 \\
\hline At4g31800 & WRKY18 & & & & & & & & & & 0 \\
\hline At1g80840 & WRKY40 & 2 & & & & & & 2 & & & 4 \\
\hline At3g19580 & $A Z F 2$ & 1 & & & & & 1 & & & & 2 \\
\hline Atlg27730 & ZAT10 & 1 & 2 & & & & & & & & 3 \\
\hline At5g59820 & ZAT12 & & & & 1 & 1 & & & 1 & 1 & 4 \\
\hline
\end{tabular}

Number of G-box and G-box like cis-elements in $1 \mathrm{kb5}$-upstream region of TFs (TAIR7_upstream_1000) was identified by using Promomer (http://bar.utoronto.ca/welcome.htm). MYC-binding affinities on G-box and G-box like motifs have been previously studied (Godoy et al., 2011). The first sequence from the left represents the canonical G-box with the highest affinity, whereas the eight other sequences represent G-box like motifs with decreasing affinity (Godoy et al., 2011).

particular GS biosynthesis and phenylpropanoid biosynthesis were affected. Since GS are known insect deterrents, the altered GSprofile observed in wrky mutants might have contributed to their enhanced susceptibility. The phenylpropanoid pathway provides precursors of various secondary metabolites related to abiotic and biotic stress, including sinapate esters, lignin, suberin, flavonols, and anthocyanins (Vogt, 2010). Polyphenols include also insect repellents like catechin, rotenone, and phaseolin (Schoonhoven et al., 2005). However these compounds are not produced in Arabidopsis and it remains still debatable whether anthocyanins or any metabolite derived from the phenylpropanoid pathway have deterrent effects against herbivores. Our observation that genes from the last steps of anthocyanins and flavonols biosynthesis are no longer induced by $S$. littoralis in wrky18wrky40 suggests that these compounds might be important for defense. It would thus be interesting to perform a targeted metabolic profiling of this mutant.

It was reported previously that WRKY18 and WRKY40 have opposite effects on resistance to necrotrophic and biotrophic pathogens. A wrky18wrky40 double mutant was more susceptible to Botrytis cinerea while it was more resistant to Pseudomonas syringae (Xu et al., 2006). Since defense to B. cinerea requires a functional JA-pathway (Rowe et al., 2010), these WRKYs might play an important role in JA-mediated responses. In addition, a recent study found that WRKY18 and WRKY40 were involved in early ABA signaling (Shang et al., 2010). Interestingly, ABA deficient mutants have been shown to be more sensitive to herbivore 
insects (Bodenhausen and Reymond, 2007). A more detailed analysis of the respective roles of GS, phenylpropanoids and/or defense proteins in the WRKY-dependent response to herbivory will be interesting in the future. In addition, WRKY60, a close homolog of WRKY18, and WRKY40, was shown to form homoand hetero-complexes with these factors and played a partially redundant role in Arabidopsis response to B. cinerea and P. syringae (Xu et al., 2006). A study of wrky18/40/60 triple mutant might unveil an increased susceptibility to $S$. littoralis herbivory and a more pronounced alteration of the transcriptome.

Although there were no marked overall differences in expression patterns of nac019, nac055, and nac019nac055 mutants compared to Col-0, these profiles were nevertheless not identical. Previously, these two TFs have been shown to be regulated by $\mathrm{MYC2}$, to form homo-and hetero-dimers and to directly control the expression of VSP1, a close homolog of VSP2 (Bu et al., 2008). Besides forming a distinct clade in the NAC protein family, NAC019, NAC055, and their homolog NAC072/RD26 have been shown to bind in vitro to the CATGTG motif (Tran et al., 2004), a G-box like motif to which MYC2, MYC3, and MYC4 also bind with high affinity (Fernández-Calvo et al., 2011). This would suggest that MYCs and NACs compete for the same binding site or form a complex. Consistent with the presence of at least two G-boxes in the promoter of NAC019 and NAC055, we found that their induction by herbivory was highly reduced in $m y c 234$, which suggests that MYC2, MYC3, and MYC4 directly regulate the expression of these genes. Indeed, a recent study showed that MYC2 binds directly to the promoter of NAC019, NAC055, and NAC072 and that these TFs positively regulate coronatinemediated suppression of the salicylic acid (SA) pathway (Zheng et al., 2012). The negative cross-talk between JA and SA is a relatively well-understood process (Pieterse et al., 2012) which could explain the insect sensitive phenotypes of nac mutants. In the presence of insects, JA might represses the SA signaling pathway via these NAC TFs, whereas elevated SA, as observed in triple nac mutants, repress the JA signaling pathway (Zheng et al., 2012). The fact that whole-genome transcription analysis did not show any major differences between nac019, nac055, nac019nac055, and Col-0 in response to herbivory might have been due to a compensatory effect of NAC072 and may have thus prevented a comprehensive characterization of the role of NACs in insect defense. Further investigations with nac019/055/072 triple mutants will be needed to determine whether NACs directly regulate insect defense genes or whether this is done indirectly by repressing the SA pathway.

ZAT12 and ZAT10 are known to play a role in plant defense to oxidative stress. It was reported that zat 12 plants are more sensitive to $\mathrm{H}_{2} \mathrm{O}_{2}$ application and are unable to activate ROS-scavenging transcripts (Rizhsky et al., 2004). In addition, overexpression of ZAT10 elevated the expression of ROS-responsive genes (Mittler et al., 2006). Since ROS production has been implicated in defense against herbivores (Kerchev et al., 2012), the enhanced susceptibility of zat10 and zat 12 mutants could be explained by a decreased ability to generate ROS. Further experiments will be required to test this hypothesis.

Finally, erf13 and rrtf1, two TF mutants that belong to the same sub-family of ERF/AP2 factors, were clustered separately from

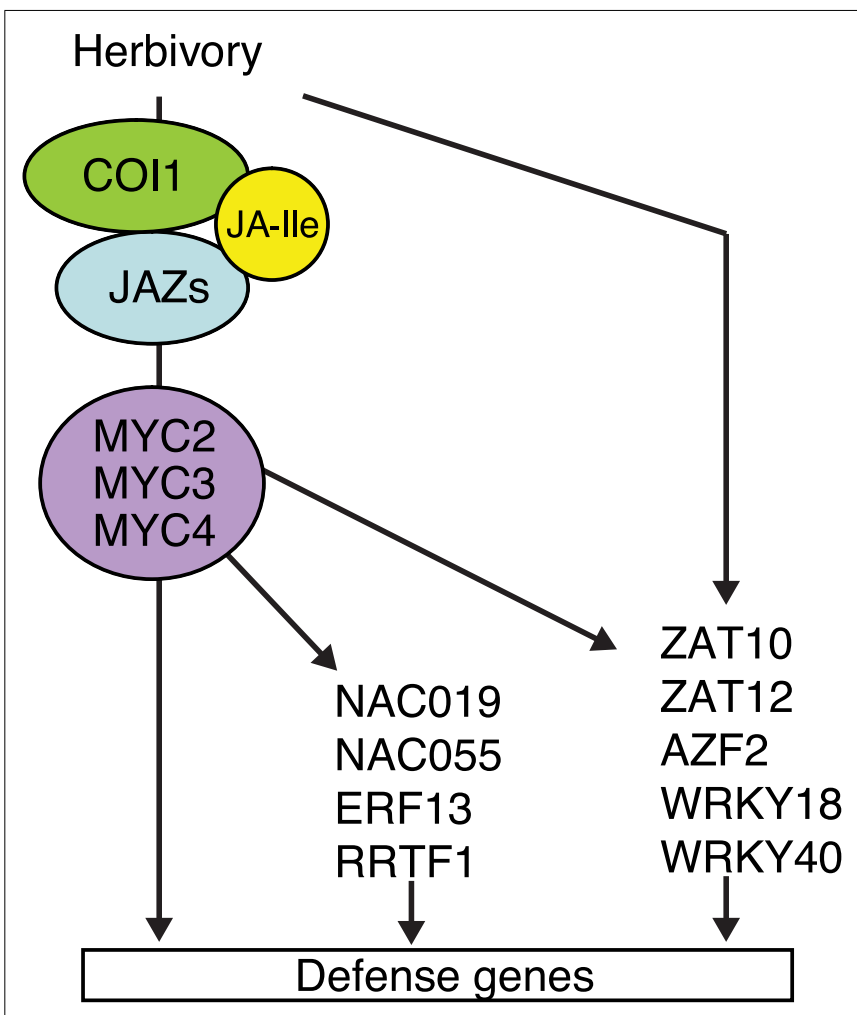

FIGURE 7 |A model for the transcriptional network in defense against chewing insects. In response to herbivory, plants produce JA-lle. This hormone is detected by its receptor COI1 that in turn degrades JAZ repressors (not shown) to allow the transcriptional activity of MYC2, MYC3, and MYC4 TFs. As a consequence, MYCs activate the expression defense genes and downstream TFs. In addition, herbivory induces the expression of several TFs, which partially depend on COI1 and MYCs. How these TFs are regulated and which are their target genes remains unknown. This model only contains TFs described in this study.

Col-0 and other TF mutants. This difference can be attributed to the fact that both mutants had a more pronounced induction of many insect-induced genes. However, since these mutants were more susceptible to $S$. littoralis herbivory, it does not seem that this enhanced expression played a significant role in their response to herbivory.

In conclusion, this study demonstrates the involvement of several novel TFs in plant defense against insects. We find that MYC2/MYC3/MYC4 are the main contributors of resistance to a generalist herbivore and that they constitute a central hub that controls the expression of downstream TFs. In addition, JAindependent factors also contribute significantly to defense. In the future, more work will be necessary to identify the complete regulatory network and associated genes that are involved in defense against insects.

\section{ACKNOWLEDGMENTS}

We thank Roland Reist (Syngenta, Stein, Switzerland) for providing S. littoralis eggs, Blaise Tissot for help in growing plants, and Caroline Gouhier-Darimont for technical support. This work was supported by the Swiss National Science Foundation. 


\section{SUPPLEMENTARY MATERIAL}

The Supplementary Material for this article can be found online at http://www.frontiersin.org/Plant-Microbe_Interaction/ 10.3389/fpls.2013.00013/abstract

\section{REFERENCES}

Baldwin, I. T. (1998). Jasmonateinduced responses are costly but benefit plants under attack in native populations. Proc. Natl. Acad. Sci. U.S.A. 95, 8113-8118.

Beekwilder, J., van Leeuwen, W., van Dam, N. M., Bertossi, M., Grandi, V., Mizzi, L., et al. (2008). The impact of the absence of aliphatic glucosinolates on insect herbivory in Arabidopsis. PLoS ONE 3:e2068. doi:10.1371/journal.pone.0002068

Bodenhausen, N., and Reymond, P. (2007). Signaling pathways controlling induced resistance to insect herbivores in Arabidopsis. Mol. Plant Microbe Interact. 20, 1406-1420.

Boter, M., Ruíz-Rivero, O., Abdeen, A., and Prat, S. (2004). Conserved MYC transcription factors play a key role in jasmonate signaling both in tomato and Arabidopsis. Genes Dev. 18, 1577-1591.

Bu, Q., Jiang, H., Li, C.-B., Zhai, Q., Zhang, J., and Wu, X. (2008). Role of the Arabidopsis thaliana NAC transcription factors ANAC019 and ANAC055 in regulating jasmonic acid-signaled defense responses. Cell Res. 18, 756-767.

Chini, A., Fonseca, S., Fernández, G., Adie, B. A. T., Chico, J. M., Lorenzo, O., et al. (2007). The JAZ family of repressors is the missing link in jasmonate signalling. Nature 448, 666-671.

de Vos, M., Denekamp, M., Dicke, M., Vuylsteke, M., Van Loon, L. C., Smeekens, S. C., et al. (2006). The Arabidopsis thaliana transcription factor AtMYB102 functions in defense against the insect herbivore Pieris rapae. Plant Signal. Behav. 1, 305-311.

de Vos, M., Van Oosten, V. R., Van Poecke, R. M. P., Van Pelt, J. A., Pozo, M. J., Mueller, M. J., et al. (2005). Signal signature and transcriptome changes of Arabidopsis during pathogen and insect attack. Mol. Plant Microbe Interact. 18, 923-937.

Devoto, A., Ellis, C., Magusin, A., Chang, H.-S., Chilcott, C., Zhu, T., et al. (2005). Expression profiling reveals COI1 to be a key regulator of genes involved in woundand methyl jasmonate-induced secondary metabolism, defence, and hormone interactions. Plant Mol. Biol. 58, 497-513.
Dombrecht, B., Xue, G. P., Sprague, S. J., Kirkegaard, J. A., Ross, J. J., Reid, J. B., et al. (2007). MYC2 differentially modulates diverse jasmonatedependent functions in Arabidopsis. Plant Cell 19, 2225-2245.

Fernández-Calvo, P., Chini, A., Fernández-Barbero, G., Chico, J. M., Gimenez-Ibanez, S., Geerinck, J., et al. (2011). The Arabidopsis bHLH transcription factors MYC3 and MYC4 are targets of JAZ repressors and act additively with MYC2 in the activation of jasmonate responses. Plant Cell 23, 701-715.

Feys, B. J., Benedetti, C. E., Penfold, C. N., and Turner, J. G. (1994). Arabidopsis mutants selected for resistance to the phytotoxin coronatine are male sterile, insensitive to methyl jasmonate, and resistant to a bacterial pathogen. Plant Cell 6, 751-759.

Fonseca, S., Chini, A., Hamberg, M., al. (2009). (+)-7-iso-Jasmonoyl-Lisoleucine is the endogenous bioactive jasmonate. Nat. Chem. Biol. 5, 344-350.

Gigolashvili, T., Berger, B., Mock, H.P., Müller, C., Weisshaar, B., and Flügge, U.-I. (2007). The transcription factor HIG1/MYB51 regulates indolic glucosinolate biosynthesis in Arabidopsis thaliana. Plant J. 50, 886-901.

Gigolashvili, T., Engqvist, M., Yatusevich, R., Müller, C., and Flügge, U.-I. (2008). HAG2/MYB76 and HAG3/MYB29 exert a specific and coordinated control on the regulation of aliphatic glucosinolate biosynthesis in Arabidopsis thaliana. New Phytol. 177, 627-642.

Glauser, G., Schweizer, F., Turlings, T. C. J., and Reymond, P. (2012). Rapid profiling of intact glucosinolates in Arabidopsis leaves by UHPLCQTOFMS using a charged surface hybrid column. Phytochem. Anal.23, 520-528.

Godoy, M., Franco-Zorrilla, J. M., Pérez-Pérez, J., Oliveros, J. C., Lorenzo, O., and Solano, R. (2011). Improved protein-binding microarrays for the identification of DNAbinding specificities of transcription factors. Plant J. 66, 700-711.

Grubb, C., and Abel, S. (2006). Glucosinolate metabolism and its control. Trends Plant Sci. 11, 89-100.

Halitschke, R., Schittko, U., Pohnert, G., Boland, W., and Baldwin, I. Adie, B., Porzel, A., Kramell, R., et

Table S1 | List of insect-induced TFs.

Table S2 | Whole-genome expression data for Col-0 and TF mutants.

\section{Table S3 | List of primers used in this study}

T. (2001). Molecular interactions between the specialist herbivore Manduca sexta (Lepidoptera, Sphingidae) and its natural host Nicotiana attenuata. III. Fatty acid-amino acid conjugates in herbivore oral secretions are necessary and sufficient for herbivore-specific plant responses. Plant Physiol. 125, 711-717.

Halkier, B. A., and Gershenzon, J. (2006). Biology and biochemistry of glucosinolates. Annu. Rev. Plant Biol. 57, 303-333.

Hirai, M. Y., Sugiyama, K., Sawada, Y., Tohge, T., Obayashi, T., Suzuki, A., et al. (2007). Omics-based identification of Arabidopsis Myb transcription factors regulating aliphatic glucosinolate biosynthesis. Proc. Natl. Acad. Sci. U.S.A. 104, 6478-6483.

Howe, G. A., and Jander, G. (2007). Plant immunity to insect herbivores. Annu. Rev. Plant Biol. 59, 41-66.

Howe, G. A., Lightner, J., Browse, J., and Ryan, C. A. (1996). An octadecanoid pathway mutant (JL5) of tomato is compromised in signaling for defense against insect attack. Plant Cell 8, 2067-2077.

Johnson, E. T., and Dowd, P. F. (2004). Differentially enhanced insect resistance, at a cost, in Arabidopsis thaliana constitutively expressing a transcription factor of defensive metabolites. J. Agric. Food Chem. 52, 5135-5138.

Kerchev, P. I., Fenton, B., Foyer, C. H., and Hancock, R. D. (2012). Plant responses to insect herbivory: interactions between photosynthesis, reactive oxygen species and hormonal signalling pathways. Plant Cell Environ. 35, 441-453.

Li, L., Zhao, Y., McCaig, B. C., Wingerd, B. A., Wang, J., Whalon, M. E., et al. (2004). The tomato homolog of CORONATINE-INSENSITIVE1 is required for the maternal control of seed maturation, jasmonatesignaled defense responses, and glandular trichome development. Plant Cell 16, 126-143.

Liechti, R., Csárdi, G., Bergmann, S., Schütz, F., Sengstag, T., Boj, S. F., et al. (2010). EuroDia: a beta-cell gene expression resource. Database (Oxford) 2010, baq024.

Liu, Y., Ahn, J.-E., Datta, S., Salzman, R. A., Moon, J., Huyghues-Despointes, B., et al. (2005). Arabidopsis vegetative storage protein is an anti-insect acid phosphatase. Plant Physiol. 139, 1545-1556.

Lorenzo, O., Chico, J. M., SánchezSerrano, J. J., and Solano, R. (2004) JASMONATE-INSENSITIVE1

encodes a MYC transcription factor essential to discriminate between different jasmonate-regulated defense responses in Arabidopsis. Plant Cell 16, 1938-1950.

Manners, J. M. J., Penninckx, I. A. I., Vermaere, K. K., Kazan, K. K., Brown, R. L. R., Morgan, A. A., et al. (1998). The promoter of the plant defensin gene PDF1.2 from Arabidopsis is systemically activated by fungal pathogens and responds to methyl jasmonate but not to salicylic acid. Plant Mol. Biol. 38, 1071-1080.

McConn, M., Creelman, R. A., Bell, E., Mullet, J. E., and Browse, J. (1997). Jasmonate is essential for insect defense in Arabidopsis. Proc. Natl. Acad. Sci. U.S.A. 94, 5473-5477.

Mewis, I., Tokuhisa, J. G., Schultz, J. C., Appel, H. M., Ulrichs, C., and Gershenzon, J. (2006). Gene expression and glucosinolate accumulation in Arabidopsis thaliana in response to generalist and specialist herbivores of different feeding guilds and the role of defense signaling pathways. Phytochemistry 67, 2450-2462.

Misra, P., Pandey, A., Tiwari, M., Chandrashekar, K., Sidhu, O. P., Asif, M. H., et al. (2010). Modulation of transcriptome and metabolome of tobacco by Arabidopsis transcription factor, AtMYB12, leads to insect resistance. Plant Physiol. 152, 2258-2268.

Mittler, R., Kim, Y., Song, L., Coutu, J., Coutu, A., Ciftci-Yilmaz, S., et al. (2006). Gain- and loss-of-function mutations in Zat 10 enhance the tolerance of plants to abiotic stress. FEBS Lett. 580, 6537-6542.

Paschold, A., Halitschke, R., and Baldwin, I. T. (2007). Co(i)ordinating defenses: NaCOI 1 mediates herbivore- induced resistance in Nicotiana attenuata and reveals the role of herbivore movement in avoiding defenses. Plant J. 51, 79-91.

Pauwels, L., Barbero, G. F., Geerinck, J., Tilleman, S., Grunewald, W., Pérez, A. C., et al. (2010). NINJA connects the co-repressor TOPLESS to jasmonate signalling. Nature 464, 788-791. 
Pauwels, L., and Goossens, A. (2011). The JAZ proteins: a crucial interface in the jasmonate signaling cascade. Plant Cell 23, 3089-3100.

Penninckx, I. A., Thomma, B. P. H. J., Buchala, A. J., Métraux, J. P., and Broekaert, W. F. (1998). Concomitant activation of jasmonate and ethylene response pathways is required for induction of a plant defensin gene in Arabidopsis. Plant Cell 10, 2103-2113.

Pieterse, C. M. J., der Does Van, D., Zamioudis, C., Leon-Reyes, A., and Van Wees, S. C. M. (2012). Hormonal modulation of plant immunity. Annu. Rev. Cell Dev. Biol. 28, 489-521.

Pré, M. M., Atallah, M. M., Champion, A. A., De Vos, M. M., Pieterse, C. M. J. C., and Memelink, J. J. (2008). The AP2/ERF domain transcription factor ORA59 integrates jasmonic acid and ethylene signals in plant defense. Plant Physiol. 147, 1347-1357.

Reymond, P., Bodenhausen, N., Van Poecke, R. M. P., Krishnamurthy, V., Dicke, M., and Farmer, E. E. (2004). A conserved transcript pattern in response to a specialist and a generalist herbivore. Plant Cell 16, 3132-3147.

Reymond, P., Weber, H., Damond, M., and Farmer, E. E. (2000). Differential gene expression in response to mechanical wounding and insect feeding in Arabidopsis. Plant Cell 12, 707-720.

Rizhsky, L., Davletova, S., Liang, H., and Mittler, R. (2004). The zinc finger protein Zat12 is required for cytosolic ascorbate peroxidase 1 expression during oxidative stress in Arabidopsis. J. Biol. Chem. 279, 11736-11743.

Rowe, H. C., Walley, J. W., Corwin, J., Chan, E. K.-F., Dehesh, K., and Kliebenstein, D. J. (2010). Deficiencies in jasmonate-mediated plant defense reveal quantitative variation in Botrytis cinerea pathogenesis. PLoS Pathog. 6:e1000861. doi:10.1371/journal.ppat.1000861
Schoonhoven, L. M., van Loon, J. J. A., and Dicke, M. (2005). Insect-Plant Biology. Oxford: Oxford University Press.

Sclep, G., Allemeersch, J., Liechti, R., De Meyer, B., Beynon, J., Bhalerao, R., et al. (2007). CATMA, a comprehensive genome-scale resource for silencing and transcript profiling of Arabidopsis genes. BMC Bioinformatics 8:400. doi:10.1186/14712105-8-400

Shang, Y., Yan, L., Liu, Z.-Q., Cao, Z., Mei, C., Xin, Q., et al. (2010). The Mg-chelatase $\mathrm{H}$ subunit of Arabidopsis antagonizes a group of WRKY transcription repressors to relieve ABA-responsive genes of inhibition. Plant Cell 22, 1909-1935.

Sheard, L. B., Tan, X., Mao, H., Withers, J., Ben-Nissan, G., Hinds, T. R., et al. (2010). Jasmonate perception by inositol-phosphatepotentiated COI1-JAZ co-receptor. Nature 468, 400-405.

Skibbe, M., Qu, N., Galis, I., and Baldwin, I. T. (2008). Induced plant defenses in the natural environment: Nicotiana attenuata WRKY3 and WRKY6 coordinate responses to herbivory. Plant Cell 20, 1984-2000.

Sønderby, I. E., Burow, M., Rowe, H. C., Kliebenstein, D. J., and Halkier, B. A. (2010). A complex interplay of three R2R3 MYB transcription factors determines the profile of aliphatic glucosinolates in Arabidopsis. Plant Physiol. 153, 348-363.

Stintzi, A., Weber, H., Reymond, P., Browse, J., and Farmer, E. E. (2001). Plant defense in the absence of jasmonic acid: the role of cyclopentenones. Proc. Natl. Acad. Sci. U.S.A. 98, 12837-12842.

Thaler, J. S., Owen, B., and Higgins, V. J. (2004). The role of the jasmonate response in plant susceptibility to diverse pathogens with a range of lifestyles. Plant Physiol. 135, 530-538.

Thines, B., Katsir, L., Melotto, M., Niu, Y., Mandaokar, A., Liu, G., et al.
(2007). JAZ repressor proteins are targets of the SCF(COI1) complex during jasmonate signalling. Nature 448, 661-665.

Thomma, B. P. H. J., Eggermont, K., and Broekaert, W. F. (2000). Disease development of several fungi on Arabidopsis can be reduced by treatment with methyl jasmonate. Plant Physiol. Biochem. 38, 421-427.

Tran, L.-S. P., Nakashima, K., Sakuma, Y., Simpson, S. D., Fujita, Y., Maruyama, K., et al. (2004). Isolation and functional analysis of Arabidopsis stress-inducible NAC transcription factors that bind to a drought-responsive cis-element in the early responsive to dehydration stress 1 promoter. Plant Cell 16 2481-2498.

Verhage, A., Vlaardingerbroek, I., Raaijmakers, C., Van Dam, N., Dicke, M. Van Wees, S. C. M., et al. (2011). Rewiring of the jasmonate signaling pathway in Arabidopsis during insect gerbivory. Front. Plant Sci. 2:47. doi:10.3389/fpls.2011.00047

Vogt, T. (2010). Phenylpropanoid biosynthesis. Mol. Plant 3, 2-20.

Walling, L. L. (2000). The myriad plant responses to herbivores. J. Plant Growth Regul. 19, 195-216.

Wittstock, U., and Gershenzon, J. (2002). Constitutive plant toxins and their role in defense against herbivores and pathogens. Curr. Opin. Plant Biol. 5, 300-307.

Xie, D., Feys, B. J., James, S., NietoRostro, M., and Turner, J. G. (1998). COI1: an Arabidopsis gene required for jasmonate-regulated defense and fertility. Science 280, 1091-1094.

Xu, X., Chen, C., Fan, B., and Chen, Z. (2006). Physical and functional interactions between pathogeninduced Arabidopsis WRKY18, WRKY40, and WRKY60 transcription factors. Plant Cell 18, 1310-1326.

Yan, Y., Stolz, S., Chételat, A., Reymond, P., Pagni, M., Dubugnon, L., et al.
(2007). A downstream mediator in the growth repression limb of the jasmonate pathway. Plant Cell 19, 2470-2483.

Zheng, X.-Y., Spivey, N. W., Zeng, W., Liu, P.-P., Fu, Z. Q., Klessig, D. F., et al. (2012). Coronatine promotes Pseudomonas syringae virulence in plants by activating a signaling cascade that inhibits salicylic acid accumulation. Cell Host Microbe 11, 587-596.

Zhu, Z., An, F., Feng, Y., Li, P., Xue, L. A. M., Jiang, Z., et al. (2011) Derepression of ethylene-stabilized transcription factors (EIN3/EIL1) mediates jasmonate and ethylene signaling synergy in Arabidopsis. Proc. Natl. Acad. Sci. U.S.A. 108, 12539-12544.

Conflict of Interest Statement: The authors declare that the research was conducted in the absence of any commercial or financial relationships that could be construed as a potential conflict of interest.

Received: 14 December 2012; paper pending published: 11 January 2013; accepted: 18 January 2013; published online: 04 February 2013.

Citation: Schweizer F, Bodenhausen N, Lassueur S, Masclaux FG and Reymond $P$ (2013) Differential contribution of transcription factors to Arabidopsis thaliana defense against Spodoptera littoralis. Front. Plant Sci. 4:13. doi: 10.3389/fpls.2013.00013

This article was submitted to Frontiers in Plant-Microbe Interaction, a specialty of Frontiers in Plant Science.

Copyright (c) 2013 Schweizer, Bodenhausen, Lassueur, Masclaux and Reymond. This is an open-access article distributed under the terms of the Creative Commons Attribution License, which permits use, distribution and reproduction in other forums, provided the original authors and source are credited and subject to any copyright notices concerning any third-party graphics etc. 